\title{
Study of extended spectrum beta lactamase producing uropathogens and their antibiotic susceptibility pattern
}

\author{
Rajeswari Pilli ${ }^{1}$, Vamsi Chakradhar Kapaganty ${ }^{2, *}$ \\ Assistant Professor, Dept. of Microbiology, NRI Institute of Medical Sciences, Visakhapatnam, Andhra Pradesh, India
}

*Corresponding Author:

Email: docchaksdegr8@gmail.com

\begin{abstract}
Background \& Objectives: Escherichia coli and Klebsiella pneumoniae are major uropathogenic isolates producing Extended spectrum beta lactamases (ESBL) which confer high resistance to common antibiotics. This study was done to know the antibiogram pattern of ESBL producing isolates from urine samples.

Materials and Methods: Culture and Antibiotic susceptibility tests (AST) were done from midstream urine samples (MSU) collected from patients. AST was done by Kirby-Bauer disc diffusion method and the isolates resistant to ceftriaxone and cefotaxime were subjected to phenotypic double disc diffusion test as per CLSI guidelines using cefotaxime (30 $\mu \mathrm{g})$ disc alone and cefotaxime plus clavulanic acid $(30 / 10 \mu \mathrm{g})$ combined disc for confirmation of ESBL production.

Results: Out of 726 urine samples, 172 showed significant growth. Among 172 isolates, most predominant isolate was Escherichia coli $(81 \%)$ and then klebsiella species(10.4\%).Total ESBL producers among these isolates were $68(39.5 \%)$. Of which 63(92.6\%) E.coli and $5(7 \%)$ Klebsiella spp. These ESBL producing E.coli showed maximum resistance to Ampicillin and sensitivity to Nitrofurantoin. Similarly Klebsiella spp. showed maximum resistance to Ampicillin and sensitivity to Amikacin. These ESBL producing E.coli \& Klebsiella spp. were also minimally resistant to Meropenem, about $14 \%$ and $20 \%$ respectively.

Conclusion: ESBL producing E.coli was high among uropathogens and most of them were highly resistant. So antibiotic sensitivity testing should be routinely done in order to prevent antibiotic resistance and treatment failure. Treatment should be followed according to the local antibiogram to specific ESBL producing isolates.
\end{abstract}

Keywords: ESBL, Antibiotic susceptibility, E. coli, cefotaxime, Meropenem, UTI.

\section{Introduction}

Antibiotic resistance is a major problem among bacterial isolates. Escherichia coli and Klebsiella pneumoniae are the major organisms causing Urinary tract infection(UTI) in the community. Other organisms causing UTI are Proteus, Pseudomonas, Salmonella, Staphylococcus aureus, Staphylococcus saprophyticus and Enterococci.

Extended spectrum beta-lactamase (ESBL) are the result of mutations in the ubiquitous class A TEM or SHV beta-lactamases. TEM-1 accounts for the majority of beta-lactamase-mediated resistance. These are mainly produced by Escherichia coli and Klebsiella pneumoniae. They are also produced rarely by Proteus species, Pseudomonas aeruginosa and other Enteobacteriaceae.

ESBLs confer various degrees of resistance of bacteria to broad spectrum cephalosporins, aztreonam and extended spectrum penicillins. These also confer resistance to other class of antibiotics which leads to development of Multi drug resistance (MDR) organisms which are resistant to more than two classes of antibiotics. Treatment for these ESBL producing isolates is becoming a challenge to the clinicians because of multidrug resistance which can also be transferred from one bacteria to the other. Carbepenem resistance is also emerging more recently among these isolates. ${ }^{1}$

ESBLs were first reported in Germany in 1983 and subsequently increasing worldwide. ${ }^{2,3}$ According to area, hospital, patients, virulence of isolate and severity of infection prevalence of ESBL producing isolates varies. So percentage of ESBLs was different in various studies. ${ }^{4}$

The occurence of ESBL producing organisms is rapidly changing with time. Their occurrence also varies from place to place. The endemicity of ESBL producers have resulted in outbreaks of infection in various hospitals worldwide. Antibiotics used against various ESBL producers leads to failures and increased patient mortality. ${ }^{5}$

Infection control and empiric choice of antibiotic mainly depends on the early detection of ESBLs. ${ }^{6}$ This study was done to know the antibiogram pattern of ESBL producing isolates from urine samples. Proper screening of ESBL positive isolates should be done and according to the local antibiogram required antibiotic should be given.

\section{Materials and Methods}

Setting: Current study was done in the Department of Microbiology, NRI Institute of Medical Sciences, sangivalasa, Visakhapatnam from $1^{\text {st }}$ April to 30th September 2017.

Specimens: Total 726 clean voided midstream samples of urine were collected in wide mouthed sterile container and transported to the laboratory. Processing of the sample should not be delayed as urine is good nutrition medium for coliforms. Sample should be refrigerated if there is any delay. 
Inclusion criteria: Patients with no use of antibiotics prior to the test.

Identification of isolates: Blood agar and Mac Conkey medium were used for culture. Loopful of urine was inoculated on these media and incubated at $37^{\circ} \mathrm{C}$ for 24 hours. Colonies were identified by Gram's Staining and biochemical reactions ${ }^{7}$. Motility done by hanging drop preparation.

Antibiotic Susceptibility Testing: Mueller Hinton Agar was used for Antibiotic Susceptibility Test which was done by Kirby Beur Disc diffusion method ${ }^{8}$. Himedia antibiotic discs used were ampicillin $(10 \mu \mathrm{g})$, amoxyclav $(30 \mu \mathrm{g}), \quad$ cefazoline $(30 \mu \mathrm{g}), \quad$ cefotaxime $(30 \mu \mathrm{g})$, ceftriaxone $(30 \mu \mathrm{g})$, ciprofloxacin $5 \mu \mathrm{g})$, cotrimoxazole $(1.25 / 23.75 \mu \mathrm{g})$, gentamycin $(10 \mu \mathrm{g})$, imipenem $(10 \mu \mathrm{g})$, nitrofurantoin $(300 \mu \mathrm{g})$, norfloxacin $(10 \mu \mathrm{g})$, piperacillin tazobactum $(100 \mu \mathrm{g}+10 \mu \mathrm{g})$, and Meropenem $(10 \mu \mathrm{g})$.

Isolates with zone of inhibition of less than $25 \mathrm{~mm}$ for ceftriaxone or less than $27 \mathrm{~mm}$ for cefotaxime were tested for ESBL confirmation by Double disc diffusion method using cefotaxime $(30 \mu \mathrm{g})$ alone and cefotaxime with clavulanic acid $(30 \mu \mathrm{g}+10 \mu \mathrm{g})$. Distance between the two discs should be $25 \mathrm{~mm}$ on Mueller Hinton agar. Zone of inhibition of $\geq 5 \mathrm{~mm}$ around cefotaxime clavulanic acid compared to cefotaxime alone confirms ESBL. (Fig. 1).

Ethical Consideration: Approval from ethics committee was not required for this study as urine samples were routinely cultured in the laboratory.

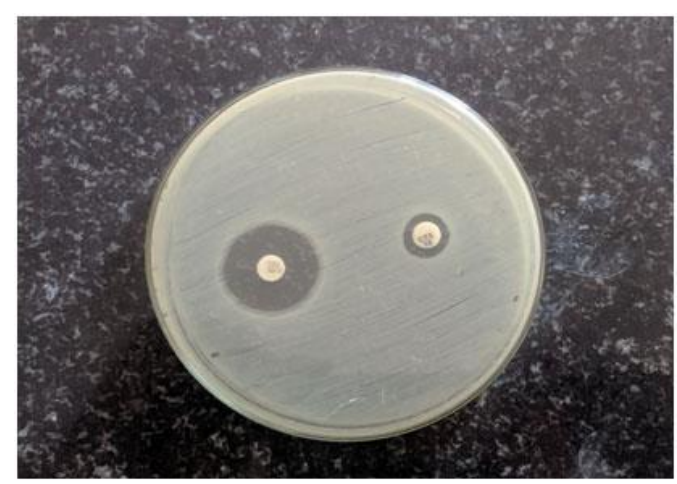

Fig. 1: Double disc diffusion method using cefotaxime \& cefotaxime clavulanic acid

\section{Results}

Total 726 urine samples were collected and tested. Among them only 172(23.6\%) samples were positive.
Out of 172 positives, $40(23.2 \%)$ were males and 132 (76.7\%) females. Male to female ratio is 1:2. Most predominant isolate among all isolates was Escherichia coli (141) and then Klebsiella species(18), Enterobacter(5), Citrobacter(3), Proteus (3) and Pseudomonas(2). (Table 1)

Among 172 isolates, 112 (65\%) were multi drug resistant (MDR). Among these 112 MDR, 103(92\%) E.coli and 9(8\%) Klebsiella spp. Total ESBL producers among these isolates were 68/172 (39.5\%). Of which 63 $(92.6 \%)$ E.coli and 5 (7\%) Klebsiella spp. (Fig. 2). Whereas Non-ESBL producers were 104/172(60.4\%), of which $78(75 \%)$ E.coli and 13(12.5\%) Klebsiella spp.

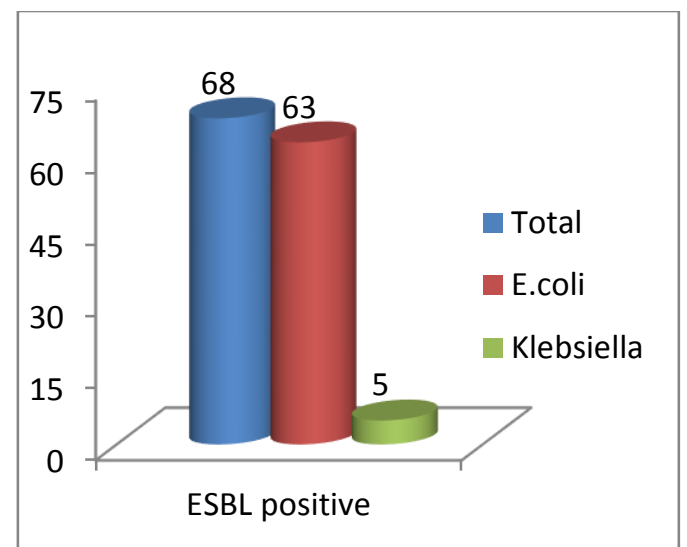

Fig. 2: Distribution of E coli and Klebsiella showing ESBL positive

Non-ESBL producing E.coli showed maximum resistance (73\%) to Ampicillin and 55\% resistance to cephalosporins and maximum sensitivity (94.8\%) to Nitrofurantoin followed by Amikacin (91\%) \& 84.6\% to Meropenem. Similarly non ESBL Klebsiella showed maximum resistance (84.6\%) to Ampicillin, $30.7 \%$ resistance to cephalosporins and maximum sensitivity $(100 \%)$ to Meropenem.

ESBL producing E.coli showed maximum 100\% resistance to Ampicillin and $100 \%$ sensitive to Nitrofurantoin. Whereas ESBL producing Klebsiella species were $100 \%$ resistant to Ampicillin, Ciprofloxacin and Amoxycillin clavulanic acid and 100\% sensitive to Amikacin. These ESBL producing E.coli \& Klebsiella species were also minimally resistant to Carbepenems ie; Meropenem about 14\% and $20 \%$ respectively. (Fig. 3) 


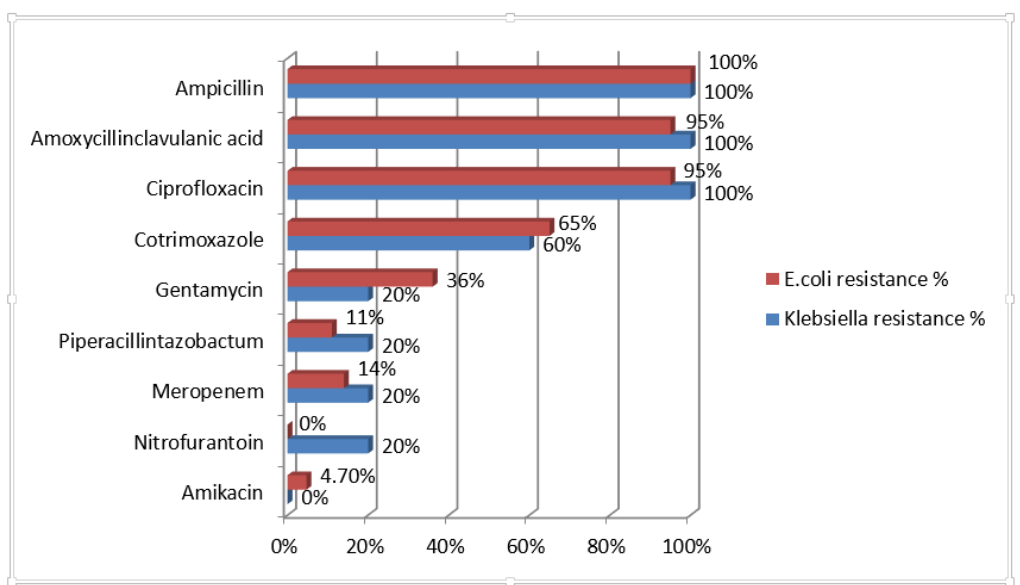

Fig. 3: Resistance pattern of ESBL producing E coli and Klebsiella

Table 1: Distribution of different Gram negative isolates in urine samples

\begin{tabular}{|l|c|c|}
\hline \multicolumn{1}{|c|}{ Organism } & Total (172) & Percentage \\
\hline E.coli & 141 & $81 \%$ \\
\hline Klebsiella & 18 & $10.4 \%$ \\
\hline Enterobacter & 5 & $2.9 \%$ \\
\hline Citrobacter & 3 & $1.7 \%$ \\
\hline Proteus & 3 & $1.7 \%$ \\
\hline Pseudomonas & 2 & $1.1 \%$ \\
\hline
\end{tabular}

Table 2: ESBL producing isolates according to age

\begin{tabular}{|c|c|c|c|}
\hline Age (years) & Total no. of isolates & ESBL positive & \% of ESBL positive \\
\hline$<15 \mathrm{yrs}$ & 9 & 3 & $33.3 \%$ \\
\hline $15-45 \mathrm{yrs}$ & 93 & 32 & $34.4 \%$ \\
\hline $46-60 \mathrm{yrs}$ & 45 & 15 & $33.3 \%$ \\
\hline$>60 \mathrm{yrs}$ & 25 & 13 & $52 \%$ \\
\hline
\end{tabular}

\section{Discussion}

Major risk factors for infection with ESBL producing organisms are long term antibiotic exposure, prolonged ICU stay, severe illness and instrumentation or catheterization. ${ }^{9}$ Inappropriate use of antibiotics resulted in the emergence of $\beta$-lactamase-producing strains that are challenge to clinical therapeutics. ${ }^{10}$ Considering the increasing rate of antibiotic resistance, the present study was done to know the antibiogram of ESBL producing E. coli and Klebsiella spp so that appropriate antibiotic can be given to the patient.

Male to female ratio is $1: 2$ correlating with Shakhya et al. ${ }^{11}$ UTI is common in females because urethra is more proximal to anus so that coliforms enter and colonize urethra and also due to short length of urethra. ${ }^{12}$

$65 \%$ of the isolates in our study were multi drug resistant (MDR) correlating with Hamed Ghadiri et $\mathrm{al}^{13}$ (65\%).One cause of the prevalence of MDR microorganisms is the production of $\beta$-lactamases such as ESBL.

In the current study a total of $39.5 \%$ were positive for ESBL production which was correlating with Yadav et al $40 \%{ }^{14}$ and nearer to kulkarni et a $1^{15} 29.16 \%$. ESBL production was highest among E.coli $92.6 \%$ followed by Klebsiella 7.3\%. High production of ESBL in E.coli was also reported by kulkarni et al. ${ }^{15}$ More than 60 years age group patients showed high prevalence of ESBL production.

ESBL producing organisms degrade extended spectrum cephalosporins and monobactams and also exhibit cross-resistance to other antibiotics, such as fluoroquinolones and aminoglycosides. ${ }^{16}$

In the current study ESBL-producing E.coli and Klebsiella isolates were highly resistant to ampicillin, amoxyclav, ciprofloxacin \& cotrimoxazole. This high resistance may be because of unwarranted use of antibiotics in the community. These findings correlating with SB Yoon et al. ${ }^{17}$

Resistance to ciprofloxacin in the current study was $95.0 \%$, which is higher than the data published by SB Yoon et $\mathrm{al}^{17} 70 \%$. Previously fluoroquinolones is the drug of choice and most frequently used medication for UTI because of their broad spectrum activity and easy administration. ${ }^{18}$ Increased resistance to these drugs occurred because of their excessive and inappropriate use.

Carbepenems, nitrofurantoin, amikacin, gentamycin and piperacillin tazobactum showed potent antibacterial activity against ESBL producing isolates. 
Among these nitrofurantoin showed maximum sensitivity to E.coli and amikacin maximum sensitivity to klebsiella. Previous studies have reported no resistance in ESBL isolates to imipenem. However, our study demonstrate the occurrence of resistance to imipenem (14\%) which correlates with Hamed Ghadiri et $\mathrm{al}^{13}(10.4 \%)$. In the current study, Aminoglycosides Amikacin \& gentamycin with resistance rates of $4.7 \%$ \& $36 \%$ to E.coli respectively, these findings lower for amikacin and higher for gentamycin than Hamed Ghadiri et $\mathrm{a}^{13}$ which showed $11 \%$ \& $15 \%$ respectively.

Amikacin use in clinical practice is limited because of its renal toxicity. Imipenem should not be considered as the empiric drug of choice even it is the most effective drug as it is the drug of last resort. It should be given only in life threatening conditions otherwise it could result in more serious challenges to the healthcare system. Nitrofurantoin or gentamycin can be given as the drug of first choice.

To limit the spread of ESBL producing isolates, ESBL detection should be included in the routine laboratory practice. Continued surveillance, appropriate use of antibiotics, and implementation of strict infection control measures are recommended to decrease ESBL frequency.

\section{Conclusion}

ESBL producing E.coli was high among uropathogens and most of them were highly resistant. So antibiotic sensitivity testing should be routinely done in order to prevent antibiotic resistance and treatment failure. Treatment should be followed according to the local antibiogram to specific ESBL producing isolates.

\section{References}

1. Rupp ME, Fey PD: Extended spectrum $\beta$-lactamase (ESBL)- producingenterobacteriaceae: considerations for diagnosis, prevention and drugtreatment. Drugs 2003,63(4):353-365.

2. Aminzadeh Z, Sadat Kashi M, Shabani M. Bacteriuria by extended-spectrum Beta-lactamase-producing Escherichia coli and Klebsiellapneumoniae: isolates in a governmental hospital in South of Tehran, Iran. Iran J Kidney Dis 2008;2(4):197-200. [PMID: 19377237]

3. Khanfar HS, Bindayna KM, Senok AC, Botta GA. Extended spectrum beta-lactamases (ESBL) in Escherichia coli and Klebsiellapneumoniae: trends in the hospital and community settings. J Infect DevCtries 2009; 3(4):295-9. [PMID: 19759493]

4. Livermore DM, Canton R, Gniadkowski M, et al. CTXM: changing the face of ESBLs in Europe. $\mathrm{J}$ AntimicrobChemother 2007; 59(2): 165-74. [http://dx.doi.org/10.1093/jac/dkl483] [PMID: 17158117].

5. Naiemi NA, Murk JL, Savelkoul PH, VandenbrouckeGrauls CM, DebetsOssenkoppYJ.Extended-spectrum beta-lactamases screening agar with AmpC inhibition. Eur J ClinMicrobiol Infect Dis. 2009;28:989-90.

6. Cohen Stuart J, Dierikx C, Al Naiemi N, Karczmarek A, Van Hoek AH, Vos P, et al. Rapid detection of TEM,
SHV and CTX-Mextended-spectrum beta-lactamases in Enterobacteriaceae using ligation-mediated amplification with microarray analysis. J AntimicrobChemother 2010;65:1377-81.

7. Collee JG, Miles RS, Watt B: Tests for identification of bacteria. In Mackie andMcCartney Practical Medical Microbiology. 14th edition. Edited by Collee JG, FraserAG, Marmion BP, Simmons A. New York: Churchill Livingstone;1996:131-149

8. Clinical and Laboratory Standards Institute (CLSI). Performance Standards for Antimicrobial Susceptibility Testing. Wayne, PA: Twenty-thi. Clinical and Laboratory Standards Institute 2013.

9. Nathisuwan S, Burgess DS, Lewis II JS, ESBLs: Epidemiology, Detection and Treatment. Pharmacotherapy2001;21(8):920-928.

10. Deshmukh DG, Damle AS, Bajaj JK, Bhakre JB, Patwardhan NS. Metallo- B-lactamase-producing clinical isolates from the patients of a tertiary care hospital. J Lab Physicians. 2011;3:93-97.

11. PoojaShakya, DhirajShrestha, ElinaMaharjan, Vijay K. Sharma and Rabin Paudyal; ESBL Production Among E. coli and Klebsiella spp. Causing Urinary Tract Infection: A Hospital Based Study; The Open Microbiology Journal, 2017,11,23-3.

12. Forbes BA, Sahm DF, Weissfeld AS. Bailey \& Scott's Diagnostic Microbiology. 12th ed. USA: Mosby Elsevier 2007.

13. HamedGhadiri, MSc, 1 Hamid Vaez, MSc, $2 *$ Kamal Razavi-Azarkhiavi, PhD; Prevalence and Antibiotic Susceptibility Patterns of Extended-Spectrum BLactamase and Metallo- $\beta$-Lactamase-Producing Uropathogenic Escherichia coli Isolates; Lab Med Fall 2014;45:290-294

14. Rajesh Kumar Yadav; Extended Spectrum BetaLactamase Producing Organisms and their Antibiotic Resistance among Study Population: A Clinical Study; International Journal of Contemporary Medical Research; Volume 3 | Issue 12 | December 2016 | ICV (2015):77.83.

15. Rugvedkulkarni, VaishaliDohe (kongre), DnyaneshwariGhadge, ArvindBhore; A study of extended spectrum betalactamase (ESBL) producers in clinical isolates; Medical Journal of Western India/February 2013/ Vol 41/ Issue 1.

16. Paterson DL, Bonomo RA; Extended-spectrum betalactamases: a clinical update. ClinMicrobiol Rev 2005;18:657-686.

17. SB Yoon, BS Lee, KD Lee, SI Hwang, HJ Lee and ZA Han; Comparison of bacterial strains and antibiotic susceptibilities in urinary isolates of spinal cord injury patients from the community and hospital; Spinal Cord (2014) 52, 298-301 \& 2014 International Spinal Cord Society All rights reserved 1362-4393/14.

18. Stamm WE, Hooton TM. Management of urinary tract infections in adults. N Engl J Med 1993;329:1328-1334. 